\title{
A new device for in vivo measurement of nasal transepithelial potential difference in cystic fibrosis patients and normal subjects
}

\author{
O. Duperrex*+, P-Y. Berclaz*+, D. Bertrand**, J.S. Lacroix ${ }^{++}$, \\ N. Pochon++, D. Belli+, T. Rochat*
}

A new device for in vivo measurement of nasal transepithelial potential difference in cystic fibrosis patients and normal subjects. O. Duperrex, P-Y. Berclaz, D. Bertrand J.S. Lacroix, N. Pochon, D. Belli, T. Rochat. (C)ERS Journals Ltd 1997.

ABSTRACT: Measurement of transepithelial potential difference (PD) on the nasal mucosa has been proposed to test for defective ion transport in cystic fibrosis $(\mathrm{CF})$, and its possible correction after gene therapy or other treatments. The "classical" method records nasal PD under the inferior turbinate, with the disadvantage that the tip of the electrode is not seen by the operator. We have developed a purposedesigned perfusion electrode for $\mathrm{PD}$ recording on the visible, medial/posterior aspect of the turbinate. We wanted to determine whether such PD recordings adequately discriminate between $\mathrm{CF}$ patients and normal subjects.

Measurements of baseline PD and response to a standardized perfusion protocol were performed in 20 normal subjects and 12 CF patients. Solutions of amiloride, with or without low chloride buffer were applied for $3 \mathrm{~min}$.

Increased baseline PD and depolarization after amiloride discriminated CF patients from normal subjects. Only one CF patient overlapped with the normal range. Superfusion of low chloride buffer with amiloride and terbutaline caused repolarization in 18 out of 20 normal subjects $(90 \%)$, consistent with physiological $\mathrm{Cl}^{-}$ secretion process, but in none of the CF patients.

We conclude that measurements of potential difference on the medial/posterior aspect of the turbinate can discriminate between cystic fibrosis patients and normal subjects. At this site, visual control of the measurement is possible. and the mucosa is easily accessible for subsequent cytological sampling or biopsy.

Eur Respir J 1997; 10: 1631-1636.
*Division of Pulmonary Medicine, Dept of Medicine, and Depts of +Paediatrics, **Physiology,${ }^{++}$Otorhinolaryngology, University Hospital, Geneva, Switzerland.

Correspondence: T. Rochat

Division de Pneumologie

Hôpital Cantonal Universitaire

$\mathrm{CH}-1211$ Genève 14

Switzerland

Keywords: Chloride

cystic fibrosis

epithelium

nose

potential difference

sodium

Received: November 251996

Accepted after revision April 121997

This study was supported by a grant from the "Société Suisse pour la Fibrose Kystique, Section Genevoise".
Measurements of transepithelial potential difference (PD) on the nasal mucosa of normal subjects and patients with cystic fibrosis (CF) were first described by KNOwLES and co-workers [1, 2] in 1981. The purpose of these measurements was to provide a method, in human subjects, for noninvasive studies on ion transport through the respiratory epithelium, which is characteristically altered in CF. In 1990, Alton et al. [3] proposed the use of measurements of nasal PD as a clinical tool for the diagnosis of $\mathrm{CF}$ when results of the sweat test are equivocal.

Recently, the development of trials of gene therapy in $\mathrm{CF}$ patients, with the aim of transferring the cystic fibrosis transmembrane conductance regulator (CFTR) complementary deoxyribonucleic acid (cDNA) into the epithelial cells of the respiratory mucosa, has opened new perspectives for the application of nasal PD measurements [4]. Because of the numerous unsolved problems in this area, the nasal mucosa has acted as an interesting surrogate for the bronchial mucosa in clinical experimentation. As compared with the bronchial mucosa, it is much easier to access, and safety problems are substantially decreased. Despite the fact that the nasal epithelium is not equivalent to the bronchial epithelium, they have many similarities, in particular the same pattern of transepithelial ion transport and the same defect in CF patients [2]. Thus, nasal PD measurements represent, for the moment, one of the best available tests to monitor the epithelial cell function in the setting of CFTR gene transfer experiments, a test that can be performed repeatedly and noninvasively.

Two recent articles have described protocols of nasal PD measurements that allow assessment of $\mathrm{Na}^{+}$absorption, as well as basal and cyclic adenosine monophosphate (cAMP)-mediated $\mathrm{Cl}^{-}$secretion by successive "superfusion" of different buffers and drugs on the nasal mucosa $[5,6]$. These protocols allow better discrimination between normal and CF-modified ion transport patterns than simple measurement of baseline PD. They have also been used to detect partial correction of the ion transport defect in CF patients after CFTR gene transfer by adenoviral vectors and by liposomes [7-9]. The authors of these protocols recommend that nasal PD measurements be performed on the floor of the nasal cavity, under the inferior turbinate, because the original work by KNOwLES and co-workers [1, 2] has shown that the epithelium of this area includes a majority (67$78 \%$ ) of ciliated epithelial cells, while the medial surface of the inferior turbinate is often the site of cuboidal metaplasia, with only $40-42 \%$ of ciliated epithelial cells 
[1]. It is also easier to stabilize the position of the perfusion catheter and exploring electrode under the turbinate than in the middle of the nasal cavity.

Measuring the nasal PD under the inferior turbinate, however, presents a drawback, in that the tip of the exploring electrode cannot be visualized during the procedure. It is, therefore, difficult to be sure that measurement is made on the precise area of the mucosa where the gene vector has been applied, that the mucosa has remained intact in this area, and that subsequent sampling by cytological brush or biopsy will be performed at the same place.

For these reasons, we considered that a thin perfusion electrode that could be placed on the medial part of the inferior turbinate, and maintained with constant visualization of the contact point with the mucosa, would be an advantage in studies of CFTR gene transfer. Cuboidal metaplasia in this area would represent a problem only if it altered the epithelial ion transport to the point that nasal PD would no longer be able to discriminate between normal and CF pattern. In addition, it is important to note that the ultimate target of treatment, namely the CF bronchial epithelium, is frequently affected by the same type of cuboidal metaplasia [10].

To address this important technical question, we devised a very thin, original, perfusion electrode, and used it to measure baseline PD in normal subjects and CF patients, on various areas of the nasal mucosa where the contact point can be easily and permanently seen by telescopic rhinoscopy. PD measurements were then performed on the medial part of the inferior turbinate, with superfusion of successive buffers and drugs, according to the protocols of KNOwLes et al. [6], and MidDLETON et al. [5]. The objective was to determine whether such measurements would discriminate between normal and CF epithelial physiology, and eventually be suitable for trials of CFTR gene transfer, or other forms of treatment of the ion transport defect of CF.

\section{Materials and methods}

\section{Subjects}

Measurements of baseline PD values were obtained in 32 normal subjects (12 females and 20 males; mean age 34 yrs, range 22-61 yrs) and $13 \mathrm{CF}$ patients ( $7 \mathrm{fe}$ males and 6 males; mean age 26 yrs, range 18-49 yrs). Superfusion studies were performed in 20 normal subjects and in $12 \mathrm{CF}$ patients. The $\mathrm{CF}$ patients all had pancreatic insufficiency, and CF diagnosis was confirmed both by clinical history and an abnormal sweat test. All participants gave informed consent and the protocol was approved by our institutional Ethics Committee.

\section{Methods}

The principle of the measurement of transepithelial nasal PD has been described in detail by others $[3,6]$. It consists in connecting an exploring electrode in contact with the nasal mucosa, and a reference electrode in contact with the subcutaneous tissue, to a high impedance voltmeter (World Precision Instruments (WPI), Berlin, Germany). A silver/silver chloride electrode (electro-cardiograph (ECG) type), taped on the volar side of the forearm after a light abrasion of the skin epithelium, was used as a reference electrode.

Our exploring electrode consisted of a Teflon-coated silver wire, $0.125 \mathrm{~mm}$ in diameter (WPI), that had been uncoated and chlorinated over the last $10 \mathrm{~mm}$ from the tip. The wire was passed into a thin intravenous catheter (outer diameter (OD) $0.63 \mathrm{~mm}$, length $30 \mathrm{~cm}$; Portex, UK) with the tip near the end of the lumen. The thin catheter passed through a rubber joint and then through a three-way connection. At the outlet of the three-way connection, the thin catheter entered a larger catheter from the same manufacturer (OD $1.34 \mathrm{~mm}$ ), which was connected by a Luer lock system. The larger, outer catheter was used for perfusion with solutions coming from the side port of the three-way connection. The length of the larger catheter was adjusted such that the thin catheter protruded a few millimetres at the extremity. The protruding tip of the thin catheter, with the silver wire inside, was slightly curved and bell-shaped by gentle heating. It was then filled with hot $4 \%$ agar that had been diluted with basal perfusion buffer. It was found that inclusion of the silver wire in an agar bridge considerably reduced the junction potential that occurred when low chloride buffer was perfused through the outer catheter. Under these conditions, the junction potential was found to be always $\leq 2 \mathrm{mV}$.

This perfusion electrode was a concentric, double lumen system, with an outside diameter of $1.34 \mathrm{~mm}$ and a slightly curved extremity, which facilitated positioning and observation of the contact point with the mucosa. Figure 1 gives a scheme of the device, and figure 2 shows the electrode in contact with the nasal mucosa, on the medial part of the inferior turbinate, as observed via a telescopic rhinoscope during measurement.

The zero offset of the electrodes was determined before and after each recording, making it possible to recognize a shift due to a defective electrode. Measurements of baseline PD were made under visual control, at four different sites: nasal septum, anterior tip, medial part and posterior part of the inferior turbinate. The values were recorded after at least $15 \mathrm{~s}$ of stable PD. Values obtained from left and right nostrils were averaged.

The perfusion electrode was then placed on the medial or slightly posterior part of the inferior turbinate. It was slowly moved until the largest PD value was obtained, together with stable recording, and constant observation of the contact point with the mucosa. The electrode was maintained in this position by attachment to an arm affixed to a helmet on the patient's head. The perfusion solutions, preheated to $37^{\circ} \mathrm{C}$ in a water bath, were administered with a peristaltic pump at a flow of $5 \mathrm{~mL}$. $\mathrm{min}^{-1}$. When switching from one perfusion to another,

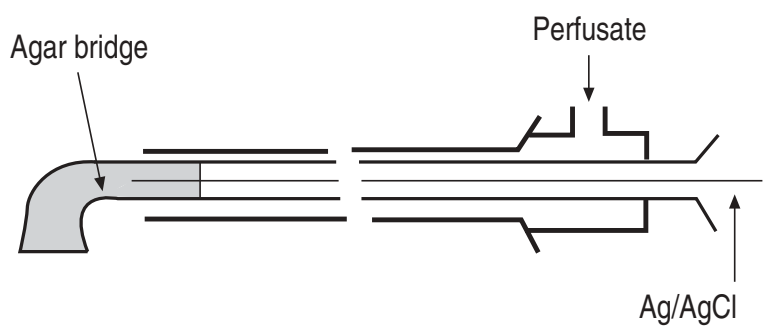

Fig. 1. - Scheme of the original perfusion electrode that was devised for the present study. Outside diameter is $1.34 \mathrm{~mm}$. See text for full description. 


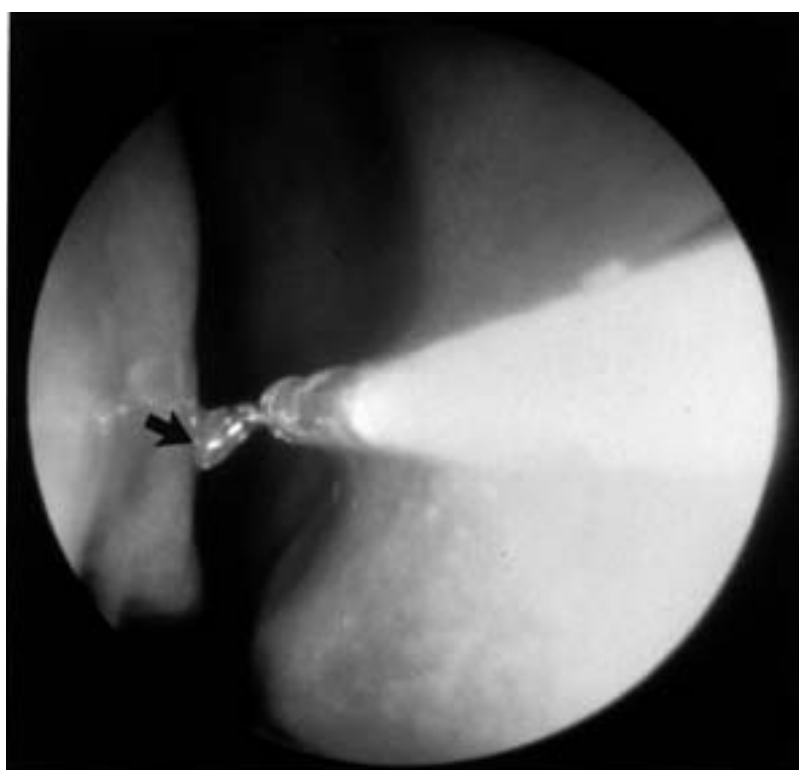

Fig. 2. - The perfusion electrode is seen by telescopic rhinoscopy. The tip, which is slightly curved to the right, is in contact with the mucosa of the medial aspect of the inferior turbinate (arrow).

the recording was interrupted for about $20 \mathrm{~s}$ whilst the tubing was purged with the new solution. In each of four CF patients, baseline nasal PD on the medial part of the inferior turbinate was obtained 5-6 times on different days. One investigator performed the measurements on three of these patients, and another investigator on the fourth. The two investigators positioned the tip of the electrode precisely on the same site of the mucosa for all measurements.

The basal buffer contained $(\mathrm{mM}): \mathrm{Na}^{+} 140, \mathrm{~K}^{+} 6$, $\mathrm{Mg}^{2+} 1, \mathrm{Ca}^{2+} 2, \mathrm{Cl}^{-} 152$, hydroxyethylpiperazine ethanesulphonic acid (HEPES) 10. A low chloride buffer was prepared by substituting $\mathrm{NaCl}$ and $\mathrm{KCl}$ with equimolar gluconate, resulting in a final $\mathrm{Cl}^{-}$concentration of 6 $\mathrm{mM}$. The $\mathrm{pH}$ of all buffers was adjusted to 7.40. Solutions were filtered and packed under sterile conditions by the pharmacist at our institution. Osmolarity and electrolyte content were checked by direct determination. Fresh stock solution of terbutaline in 4\% ascorbic acid, and amiloride in water, were prepared less than 4 $\mathrm{h}$ before the procedure.

\section{Perfusion protocol}

After stabilization of the baseline PD under superfusion of basal buffer for at least $1 \mathrm{~min}$ (Phase 1), the solutions were applied in the following order: amiloride $100 \mu \mathrm{M}$ in basal buffer until stabilization of the new baseline (Phase 2); amiloride $100 \mu \mathrm{M}$ in low chloride buffer for at least 3 min (Phase 3); amiloride $100 \mu \mathrm{M}$ + terbutaline $10 \mu \mathrm{M}$ in low chloride buffer for another 3 min (Phase 4).

\section{Statistical analysis}

Results are indicated as mean \pm SEM. Unpaired t-test was used for comparison between normal subjects and $\mathrm{CF}$ patients and differences were considered significant at p-values less than 0.05 .

\section{Results}

Figure 3 shows the results of baseline PD obtained at four different sites in normal subjects and in patients with CF. On the medial and posterior part of the inferior turbinate, baseline PD clearly differentiated normal subjects from CF patients, with very little overlap (medial part $-10.3 \pm 1.2$ versus $-31.0 \pm 2.7 \mathrm{mV}$ in normals and $\mathrm{CF}$ patients, respectively $(\mathrm{p}=0.0001)$; posterior part

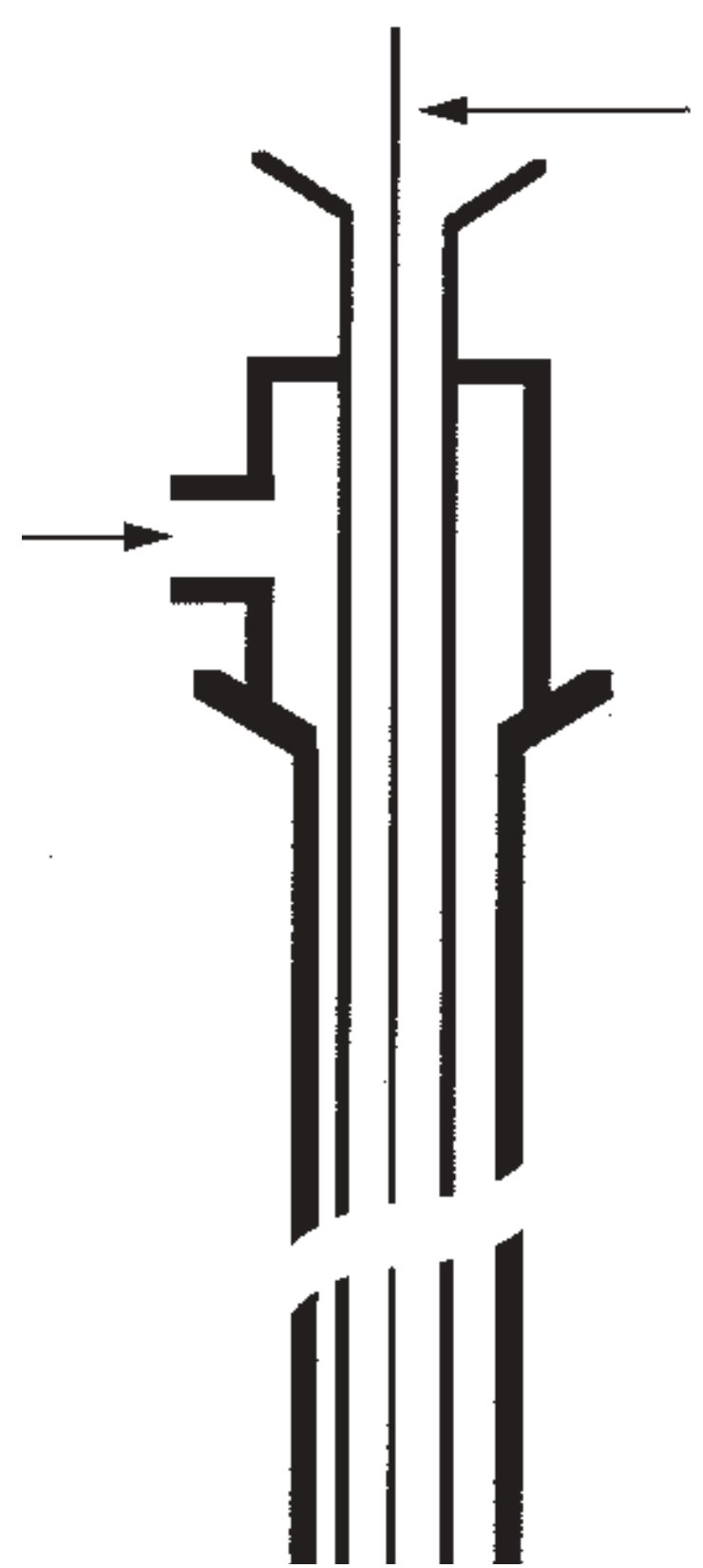

Fig. 3. - Baseline values for nasal transepithelial potential difference (PD) at various sites of the nasal mucosa where direct visualization of the contact point of the electrode is possible. a) nasal septum; b) anterior part of the inferior turbinate; c) medial part of the inferior turbinate; d) posterior part of the inferior turbinate. $\bullet$ normal subjects; $O$ : cystic fibrosis $(\mathrm{CF})$ patients. PD values are significantly different between groups for the medial and for the posterior part of the inferior turbinate $(\mathrm{p}=0.0001)$. There were 32 normal subjects, except for the posterior part of the inferior turbinate where only 16 normal subjects were tested versus $13 \mathrm{CF}$ patients. 
$-3.2 \pm 0.9$ versus $-38.9 \pm 1.6 \mathrm{mV}$, respectively; $\mathrm{p}=0.0001$ ). By contrast, there was no difference between the two groups for measurements on the nasal septum and on the anterior tip of the inferior turbinate. Left and right sides gave similar values within the same individual, with a mean difference of $3.7 \pm 0.4 \mathrm{mV}$. The coefficient of variation (sD/mean) for baseline nasal PD on the medial part of the inferior turbinate obtained in each of the four CF patients measured 5-6 times was 0.26, 0.27, 0.27 and 0.25 , respectively. Thus the variability appeared to be very constant from one subject to the other.

At the start of the perfusion protocol, the exploring electrode was placed at the site of the most negative baseline PD which, depending on the case, was on the medial or slightly posterior part of the inferior turbinate. Baseline values averaged $-15.1 \pm 1.8 \mathrm{mV}$ for normal subjects, and $-42.9 \pm 4.7 \mathrm{mV}$ for $\mathrm{CF}$ patients. The $95 \%$ confidence interval $(95 \% \mathrm{CI})$ for normal subjects (mean \pm $2 \mathrm{SD}$ ) ranged from +1 to $-32 \mathrm{mV}$. Only one $\mathrm{CF}$ patient repeatedly had baseline values in this range $(-20 \mathrm{mV})$.

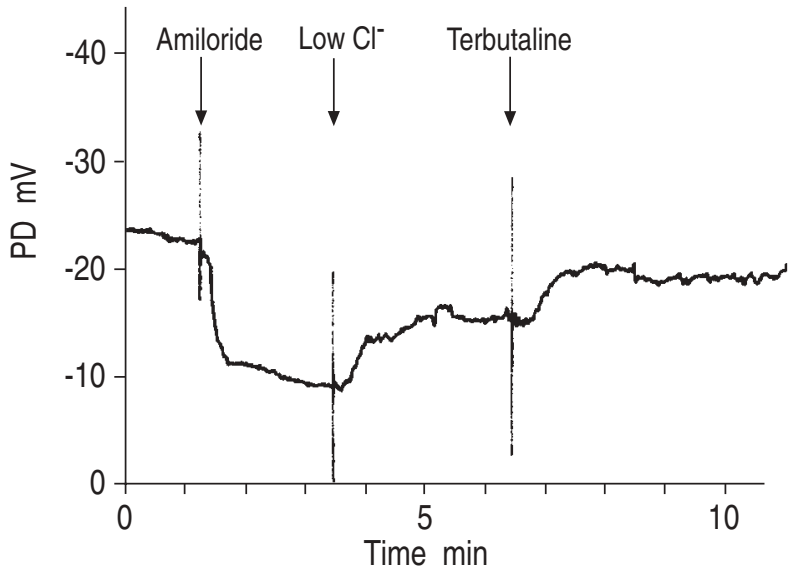

Fig. 4. - Chart recording of nasal transepithelial potential difference (PD) against time in a normal subject during the perfusion protocol. After depolarization by amiloride $(100 \mu \mathrm{M})$, two successive phases of repolarization occurred after superfusion of low chloride buffer $(6 \mathrm{mM})$ followed by terbutaline $(10 \mu \mathrm{M})$.

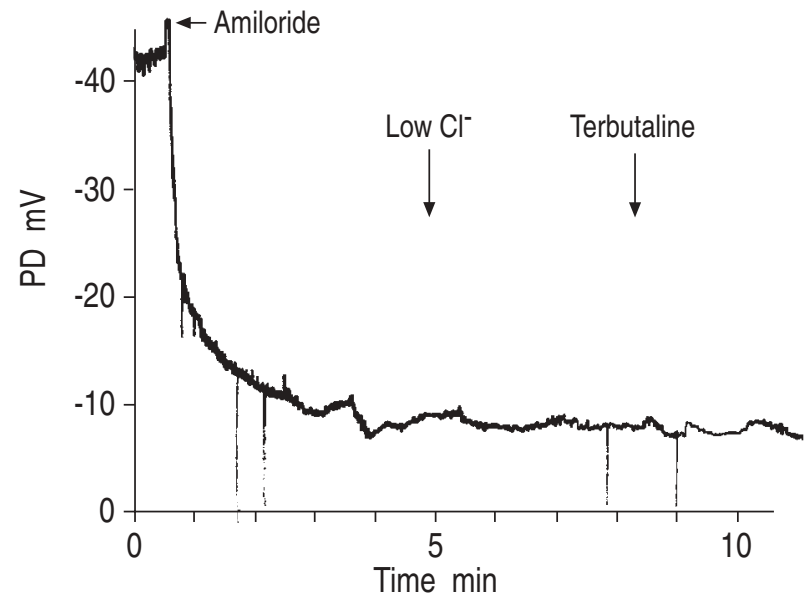

Fig. 5. - Chart recording of nasal transepithelial potential difference (PD) against time in a patient with cystic fibrosis (CF) during the perfusion protocol. After a profound depolarization caused by amiloride, superfusion of low chloride buffer and terbutaline did not cause repolarization (concentrations identical to figure 4). This indicates that no significant chloride secretion occurred in response to increased electrochemical gradient, nor to stimulation by cyclic adenosine monophosphate (cAMP).
Responses of nasal PD to the perfusion protocol are illustrated by two representative cases in figures 4 and 5. Amiloride induced a marked depolarization in both cases. Whilst low chloride buffer and terbutaline induced two successive repolarizations in the normal subject (fig. 4), these manoeuvres did not cause any persistent change of nasal PD in the CF patient (fig. 5).

The individual responses obtained in the group of normal subjects and in the group of CF patients are compared in figure 6. Amiloride caused more pronounced depolarization among $\mathrm{CF}$ patients than among normal
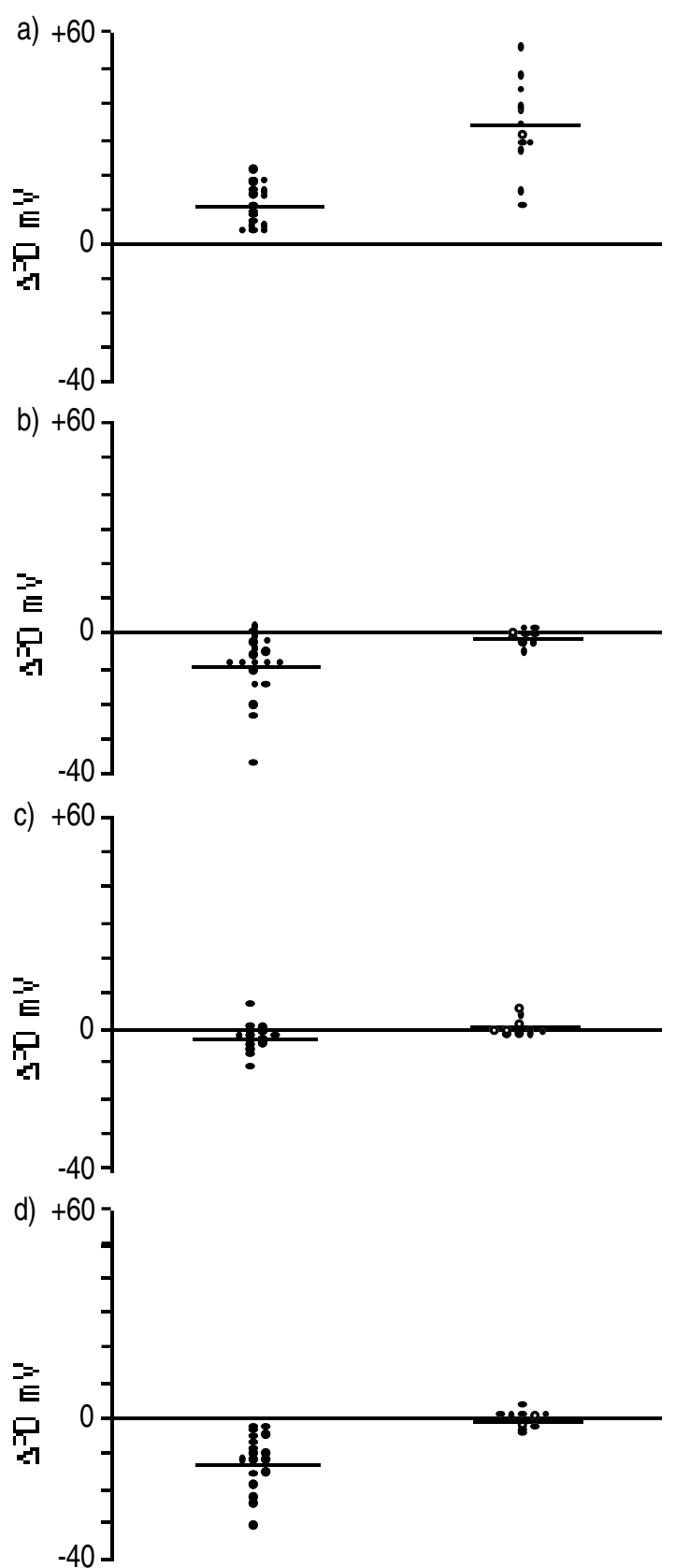

Fig. 6. - Changes in nasal transepithelial potential difference $(\triangle P D)$ after superfusion of: a) amiloride $(100 \mu \mathrm{M})$; b) low chloride buffer $(6 \mathrm{mM}) ; \mathrm{c})$ terbutaline $(10 \mu \mathrm{M})$; and d) the combined effect of low chloride + terbutaline. $\bullet$ : normal subjects; $\bigcirc$ : cystic fibrosis $(\mathrm{CF})$ patients. Changes in PD are significantly different between groups for amiloride $(\mathrm{p}=0.0001)$, low chloride $(\mathrm{p}=0.002)$, terbutaline $(\mathrm{p}=0.002)$, and the combined effect of low chloride + terbutaline $(p=0.0001)$. There were 20 normal subjects versus $12 \mathrm{CF}$ patients. 
subjects $(\triangle \mathrm{PD}$ after amiloride $=10.1 \pm 1.2$ versus $32.4 \pm 3.7$ $\mathrm{mV}$ for normal subjects and $\mathrm{CF}$ patients, respectively; $\mathrm{p}=0.0001$ ). However, when expressed as percentage of baseline, the changes were not statistically different between the two groups $(66 \pm 6 \%$ versus $76 \pm 5 \%$, respectively; NS).

Perfusion of low chloride buffer with amiloride caused definite repolarization $(\triangle P D \geq 5 \mathrm{mV})$ in 15 out of 20 normal subjects, while $\mathrm{CF}$ patients displayed no or only minimal repolarization (fig. 6). The difference between the two groups was significant $(\triangle \mathrm{PD}$ after low chloride $=-10.0 \pm 2.0$ versus $-1.3 \pm 0.5 \mathrm{mV}$ for normal subjects and $\mathrm{CF}$ patients, respectively; $\mathrm{p}=0.002$ ). The presence of terbutaline caused additional repolarization in only 7 out of 20 normal subjects, whilst CF patients did not demonstrate repolarization (fig. 6). The difference between the two groups was again significant $(\triangle P D$ after terbutaline $=3.2 \pm 0.8$ versus $+0.7 \pm 0.5 \mathrm{mV}$, respectively; $p=0.002$ ). Finally, the combined effect of low chloride buffer and terbutaline induced definite repolarization in 18 out of 20 normal subjects $(90 \%)$, but in none of the CF patients (fig. 6). The difference between the two groups was highly significant $(\triangle \mathrm{PD}$ after low chloride + terbutaline $=-13.2 \pm 1.8$ versus $-0.7 \pm 0.6$ for normal subjects and $\mathrm{CF}$ patients, respectively; $\mathrm{p}=0.0001$ ).

\section{Discussion}

The aim of this work was to set up a fast, and noninvasive method to assess abnormal ion transport of the respiratory epithelium in $\mathrm{CF}$ patients. For this purpose, we developed a new recording electrode allowing stable investigation of nasal mucosa transepithelial potential on the medial aspect of the inferior turbinate. This site of the mucosa is easily accessible for inspection and tissue sampling, if necessary. We found that normal subjects and CF patients differed markedly in their baseline PD, as well as in their response to superfusion of amiloride, low chloride, and terbutaline. These results are in accordance with those obtained on the floor of the nasal cavity, under the turbinate, by MiDDLETON et al. [5], and by KNowles et al. [6], who used a similar perfusion protocol. However, their measurements were made on a site that is not accessible, for the most part, to visual control, and which necessitates local anaesthesia for biopsy or cytological brushing.

The superfusion protocol that we applied has been used with minor differences by various authors $[5,6,8$, 9]. It was designed for detection of two characteristic abnormalities of epithelial ion transport in CF patients. Firstly, the excessive $\mathrm{Na}^{+}$absorption through the epithelium, from the lumen to the submucosa; and secondly, the failure to secrete chloride in the opposite direction, following stimulation by cAMP, under an appropriate electrochemical gradient.

Under basal conditions, transepithelial PD is due mainly to continuous absorption of $\mathrm{Na}^{+}$from the lumen [11]. Superfusion of amiloride causes the blockade of $\mathrm{Na}^{+}$channels at the apical membrane of epithelial cells, and therefore causes depolarization of the transepithelial potential. In $\mathrm{CF}$, as a consequence of the defective CFTR molecule, which contributes to the regulation of $\mathrm{Na}^{+}$channels [12], there is increased basal absorption of $\mathrm{Na}^{+}$. This is the reason for increased nasal PD under basal conditions, and for a larger decrease than normal under superfusion of amiloride.

The mean baseline PD that we observed at the medial or posterior part of the inferior turbinate, among normal subjects $(-13.9 \mathrm{mV})$ and $\mathrm{CF}$ patients $(-41.5 \mathrm{mV})$, was in the same range as that reported by Alton et al. [3], who performed their measurements along the floor of the nasal cavity $(-19.0 \mathrm{mV}$ for normal subjects and $-46.1 \mathrm{mV}$ for CF patients). We found very little overlap between the two groups, with only one $\mathrm{CF}$ value within the $95 \%$ CI of normal subjects. Finally, the mean difference that we observed between both nasal cavities within the same individuals $(3.5 \mathrm{mV})$ was similar to that reported by Alton et al. [3] (3.6 mV).

Superfusion of amiloride caused a larger depolarization in all CF patients than in normal subjects, with the exception of only one patient, who also had baseline PD in the normal range. This large, amiloride-induced depolarization is in agreement with the concept that increased baseline PD in CF patients is essentially due to excessive sodium reabsorption.

As pointed out by KNOWLES and co-workers [1, 6], it is important to realize that inflammation and light trauma may decrease the transepithelial electrical resistance, which in turn will decrease baseline PD and the absolute effect of amiloride, without affecting the process of sodium hyperabsorption. Inflammation and/or epithelial damage is probably the best explanation for the low baseline PD, with low response to amiloride, that we found in one CF patient in the present study. This observation also stresses the fact that changes in baseline $\mathrm{PD}$, and in the response to amiloride, in gene therapy or pharmacological trials aimed at improving ion transport, must be interpreted with caution because of the difficulty of ruling out an effect on transepithelial resistance in each case.

The other objective of the perfusion protocol was the assessment of the chloride secretion process across the epithelium. By blocking $\mathrm{Na}^{+}$absorption, amiloride creates an electrical gradient that favours secretion of $\mathrm{Cl}^{-}$ into the lumen. The addition of low chloride buffer on the mucosa adds a chemical gradient. This will translate into chloride secretion, and a repolarization of the transepithelial PD in subjects whose epithelia have normal chloride permeability $[5,6]$. The addition of a cAMP stimulator, such as terbutaline or isoproterenol, will open new chloride channels at the apical membrane of epithelial cells, allowing increased passage of $\mathrm{Cl}^{-}$into the lumen, and causing additional repolarization of transepithelial PD in normal subjects [5,6]. The CFTR molecule has been shown to be a cAMP-responsive chloride channel, but also a cAMP-dependent regulator of other types of chloride channels that are present at the apical membrane of respiratory epithelial cells [13]. The defective CFTR molecule in CF will be the cause of the reduced ability to secrete $\mathrm{Cl}^{-}$in response to a favourable electrochemical gradient, and of the lack of response upon cAMP stimulation.

We found that marked repolarization (arbitrarily defined by a change of PD of $\geq 5 \mathrm{mV}$ ) occurred in 18 out of 20 normal subjects $(90 \%)$ under the combined effect of low chloride buffer $\left(\mathrm{Cl}^{-} 6 \mathrm{mM}\right)$ and terbutaline $(10$ $\mu \mathrm{M})$. By contrast, none of the $12 \mathrm{CF}$ patients responded 
to the same stimulation by a repolarization of more than $-4 \mathrm{mV}$, most of them showing no change of PD at all.

We compared our results with those reported by KNOWLES and co-workers [6] who studied the combined effect of amiloride, low chloride and isoproterenol, in 20 studies performed in 17 normal subjects. Repolarization averaged about $30 \mathrm{mV}$ in their subjects, whilst we observed a mean of $13 \mathrm{mV}$ in ours. The difference could be related to a difference in potency of isoproterenol and terbutaline, both being used at $10 \mu \mathrm{M}$ concentration. We are not aware of studies comparing the two drugs under these precise conditions. Despite this limitation, the perfusion protocol that we applied on this site of the mucosa was able to discriminate between normal subjects and CF patients.

As opposed to the medial aspect of the inferior turbinate, measurements of baseline PD on the anterior tip of the turbinate and on the mucosa of the nasal septum did not discriminate between normal subjects and $\mathrm{CF}$ patients. The mucosa at the anterior tip is covered by a stratified squamous epithelium, while the mucosa of the septum contains predominantly goblet cells, with very few ciliated cells [1]. For this reason, we did not apply the perfusion protocol on these sites.

In summary, the present data indicate that measurement of nasal potential difference with a perfusion protocol, on the visible, medial or posterior part of the inferior turbinate, allowed good discrimination between normal subjects and patients with cystic fibrosis. Measurement of nasal potential difference has been shown to be of diagnostic value when patients are suspected to have some clinical form of cystic fibrosis, but have sweat chloride values in the normal range [14]. The method that we are describing here is not intended to replace the "classical" method, with measurements under the inferior turbinate, when the objective is focused on such a diagnostic purpose. Rather, it offers a more suitable approach to evaluate the effect of new forms of treatment that have recently been proposed to correct the functional defect of ion transport in cystic fibrosis. These include gene therapy, with viral and nonviral vectors, but also pharmacological treatments, for example with "chemical chaperones" [15], or compounds such as phenylbutyrate [16], which have been shown in vitro to restore the function of mutated cystic fibrosis transmembrane conductance regulator. In clinical trials aimed at evaluating such substances, the assessment of ion transport of the respiratory mucosa would be best performed on a site that is easily visible, and accessible for tissue sampling, with no (or minimal) manipulation. The method that we have presented here could satisfy these criteria.

\section{References}

1. Knowles MR, Carson JL, Collier AM, Gatzy JT, Boucher RC. Measurements of nasal transepithelial electric potential differences in normal human subjects in vivo. Am Rev Respir Dis 1981; 124: 484-490.

2. Knowles M, Gatzy J, Boucher R. Increased bioelectric potential difference across respiratory epithelia in cystic fibrosis. N Engl J Med 1981; 305: 1489-1495.

3. Alton EWFW, Currie D, Logan-Sinclair R, Warner JO, Hodson ME, Geddes DM. Nasal potential difference: a clinical diagnostic test for cystic fibrosis. Eur Respir $J$ 1990; 3: 922-926.

4. Chinet TC. Use of in vivo nasal transepithelial potential difference to evaluate efficacy in CF gene therapy phase I trials. Eur Respir J 1994; 7: 1917-1920.

5. Middleton PG, Geddes DM, Alton EWFW. Protocols for in vivo measurement of the ion transport defects in cystic fibrosis nasal epithelium. Eur Respir J 1994; 7: 2050-2056.

6. Knowles MR, Paradiso AM, Boucher RC. In vivo nasal potential difference: techniques and protocol for assessing efficacy of gene transfer in cystic fibrosis. Hum Gene Ther 1995; 6: 445-455.

7. Caplen NJ, Alton EWFW, Middleton PG, et al. Liposome-mediated CFTR gene transfer to the nasal epithelium of patients with cystic fibrosis. Nat Med 1995; 1: $39-46$.

8. Hay JG, McElvaney NG, Herena J, Crystal RG. Modification of nasal epithelial potential differences of individuals with cystic fibrosis consequent to local administration of a normal CFTR cDNA adenovirus gene transfer vector. Hum Gene Ther 1995; 6: 1487-1496.

9. Zabner J, Ramsey BW, Meeker DP, et al. Repeat administration of an adenovirus vector encoding cystic fibrosis transmembrane conductance regulator to the nasal epithelium of patients with cystic fibrosis. J Clin Invest 1996; 97: 1504-1511.

10. Sturgess JM. Ciliated cells of the lung. In: Massaro D, ed. Lung Cell Biology. New York, Marcel Dekker Inc., 1989; pp. 115-151.

11. Knowles M, Gatzy J, Boucher R. Relative ion permeability of normal and cystic fibrosis nasal epithelium. $J$ Clin Invest 1983; 71: 1410-1417.

12. Stutts MJ, Canessa CM, Olsen JC, et al. CFTR as a cAMP-dependent regulator of sodium channel. Science 1995; 269: 847-850.

13. Egan M, Flotte $\mathrm{T}$, Afione $\mathrm{S}$, et al. Defective regulation of outwardly rectifying chloride channels by protein kinase A corrected by insertion of CFTR. Nature 1992; 358: 581-584.

14. Stewart B, Zabner J, Shuber AP, Welsh MJ, McCray PB. Normal sweat chloride values do not exclude the diagnosis of cystic fibrosis. Am J Respir Crit Care Med 1995; 151: 899-903.

15. Welch WJ, Brown CR, Brown LQ, et al. Chemical chaperones correct protein folding abnormalities. Pediatr Pulmonol 1996; Suppl. 13: 258.

16. Rubenstein RC, Brusilow SW, Hamosh A, Zeitlin PL. Clinical trials of 4-phenylbutyrate for correction of sweat duct abnormalities in $\Delta \mathrm{F} 508$ homozygous cystic fibrosis patients. Pediatr Pulmonol 1996; Suppl. 13: 259.
Acknowledgements: The authors are indebted to L. Pizurki, and to W. Griffith, M. Pahud and F. Correia for technical assistance, and fruitful suggestions. 